\title{
Religiöse Individualisierung statt Säkularisierung? Eine falsche Alternative
}

\section{Antwort auf die Replik von Wohlrab-Sahr und Krüggeler}

\author{
Detlef Pollack* und Gert Pickel* \\ Europa-Universität Viadrina Frankfurt (Oder), Postfach 1786, D-15207 Frankfurt (Oder)
}

1. Zentral für die hier geführte Kontroverse ist zweifellos die Bestimmung des Individualisierungsbegriffs. Es scheint, als ob Wohlrab-Sahr und Krüggeler uns unterstellen wollten, dass wir nicht in der Lage seien, Individualisierung als einen sozialstrukturell bestimmten Prozess zu erfassen. Schon die Überschrift ihrer Replik „Strukturelle Individualisierung versus autonome Menschen " legt diese Lesart nahe. Und auch sonst heben sie immer wieder darauf ab, dass wir nur auf der personalen Ebene argumentierten, während sie auch die sozialstrukturelle und institutionelle Ebene im Auge hätten, wenn sie von Individualisierung sprechen. Eine solche Unterstellung verwundert, sind wir doch ausführlich auf die sozialstrukturellen und kulturellen Voraussetzungen von Individualisierungsprozessen eingegangen und haben wir uns doch explizit von einer Position abgegrenzt, die Individualisierung als Emanzipation des Individuums von der Gesellschaft missversteht (Pollack/Pickel 1999a: 467f.). Die sozialstrukturellen Bedingungen von Individualisierung haben wir wie andere (Beck 1983; Kohli 1985) im Ausbau des Sozialstaats, in der Erhöhung des Wohlstandsniveaus, in einer Verschiebung im Verhältnis von Arbeit und Freizeit usw. gesehen, die

\footnotetext{
* Wir danken Jörg Jacobs und Olaf Müller, beide Frankfurt (Oder), für wertvolle kritische Hinweise.
}

kulturellen Bedingungen in der Abschwächung der Gültigkeit von sozialen Normen und Werten sowie in ihrer damit einhergehenden Pluralisierung. Den kulturellen Voraussetzungen der Individualisierung, auf die Wohlrab-Sahr und Krüggeler nicht eingehen, haben wir sogar ein eigenes Kapitel gewidmet („Zwischenüberlegung“, Pollack/Pickel 1999a: 472f.). Hinsichtlich der Fassung von Individualisierung als sozialstrukturell bedingtem Prozess befinden wir uns also nicht im Dissens.

Der Aufweis sozialstruktureller und kultureller Voraussetzungen von Individualisierung ist nun allerdings nicht gleichbedeutend mit der Aussage, dass Individualisierung nichts anderes ist als die Determination des Individuums durch sozialstrukturelle und kulturelle Umstände. Ein solches Verständnis des Verhältnisses von Individuum und Gesellschaft, das dem Einzelnen gewissermaßen keinerlei Spielraum mehr ließe, würde den Begriff der Individualisierung überflüssig machen. Genau an dieser Stelle treffen wir jedoch auf eine Unschärfe in der Argumentation von Wohlrab-Sahr und Krüggeler. Einerseits definieren sie Individualisierung als „Akzeptanz" bzw. „Verinnerlichung “ eines gesellschaftlich vorgegebenen Deutungsmusters, in deren Konsequenz sich das Individuum Handlungen selbst kausal attribuiert (vgl. Wohlrab-Sahr 1997: 28). Auf der anderen Seite verstehen sie Individualisie- 
rung als „Auflösung institutioneller Verbindlichkeit zugunsten individueller Entscheidungsspielräume“ oder als „,Privatisierung des Entscheidens““. Privatisierung des Entscheidens meint aber doch wohl, dass die Entscheidung nicht gesellschaftlich vorgegeben ist - strukturell oder kulturell -, sondern individuell getroffen wird. Übernahme eines gesellschaftlichen Deutungsmusters meint hingegen, dass die Interpretation des Handelns außengeleitet erfolgt. Was wollen die Autoren nun unter Individualisierung verstehen: einen Prozess der zunehmenden Fremdbestimmung oder einen der zunehmenden Selbstbestimmung? Wohlrab-Sahr und Krüggeler partizipieren hier an den Unklarheiten und Widersprüchlichkeiten, die sich in der Diskussion der Individualisierungsthese - nicht zuletzt bei Beck selbst - immer wieder finden.

Diese Widersprüchlichkeiten machen es schwer, Individualisierung empirisch zu messen. Unser Vorschlag an dieser Stelle wäre, genauer zwischen den sozialstrukturellen Bedingungen von Individualisierung und dem Prozess der Individualisierung selbst zu unterscheiden. Auch wenn das Individuum seine Handlungsspielräume nicht einfach selbst bestimmen kann, ist es doch nur dann sinnvoll, von Individualisierung zu sprechen, wenn das Handeln und Erleben des Individuums nicht lediglich in einer Übernahme gesellschaftlicher Vorgaben besteht. In diesem Sinne haben wir Individualisierung als $z u$ nehmende Selbstbestimmung des Individuums definiert (von Autonomie ist in unserem Text nirgends die Rede). Übrigens definiert auch Ulrich Beck Individualisierung so: „Individualisierung bedeutet (...), dass die Biografie der Menschen aus vorgegebenen Fixierungen herausgelöst, offen, entscheidungsabhängig und als Aufgabe in das individuelle Handeln jedes Einzelnen gelegt wird. Die Anteile der prinzipiell entscheidungsverschlossenen Lebensmöglichkeiten nehmen ab, und die Anteile der entscheidungsoffenen, selbst herzustellenden Biografie nehmen zu. “ (Beck 1983: 58) Dies entspricht genau dem von uns verwendeten Begriff von Individualisierung. Die Akzeptanz und Verinnerlichung eines gesellschaftlichen Modus der Zurechnung, wie Wohlrab-Sahr und Krüggeler Individualisierung (unter anderem) definieren, bedeutet hingegen keine Zunahme der Entscheidungsmöglichkeiten des Individuums über sein eigenes Leben, sondern stellt eine Form der individuellen Fremdbestimmung dar. Selbstverständlich muss man sich, wenn man das kollektive Deutungsmuster der individuellen Kausalattribuierung des Handelns akzeptiert, nicht über sich selbst täuschen. Die individuellen Handlungsspielräume sind möglicherweise tatsächlich größer geworden. Doch ob das so ist, hängt nicht von der individuellen Selbstzurechnung der Handlungen ab. Und deshalb kann diese Form der Selbstzurechnung nicht konstitutiv für die Definition von Individualisierung sein.

2. Unverständlich ist uns auch, warum WohlrabSahr und Krüggeler unsere Analysen auf die Kritik des Ansatzes von Thomas Luckmann festlegen wollen. Warum wollen sie uns zum Beispiel auf die Widerlegung eines Religionsbegriffes verpflichten, von dem sie selbst sagen, dass er „unempirisch“ ist? Uns kam es jedenfalls nicht darauf an, die Theorie Luckmanns zu widerlegen. Vielmehr ging es uns darum, die Individualisierungsthese, die sich in der deutschen Religionssoziologie einer immer größer werdenden Beliebtheit erfreut und für die Luckmanns Ansatz nur einen Ausgangspunkt darstellt, der empirischen Überprüfung auszusetzen. Diese These besteht nicht nur in der bekannten Unterscheidung einer institutionell spezifischen und einer allgemeinen Sozialform von Religion, die es erlaubt, den Bedeutungsrückgang der christlichen Kirchen von Entwicklungsprozessen der Religion insgesamt abzuheben; sie hat darüber hinaus noch zwei weitere Elemente. Die Individualisierungsthese ist explizit gegen die Säkularisierungsthese gebaut und mit der hoch anspruchsvollen Aussage versehen, dass es in der Gegenwart nicht zu einem Rückgang an Religion, sondern lediglich zu Positionsverlusten der Kirchen bei gleichzeitigem Aufschwung nicht-institutionalisierter Religionsformen, also nur zu einem Formenwandel des Religiösen komme (vgl. Tyrell 1996: 445f.). Die Individualisierungsthese geht mithin von einer Art Null-Summenspiel auf dem religiösen Feld aus. Außerdem ist die Individualisierungsthese mit der Aussage verbunden, dass die De-Institutionalisierung von Religion zu ihrer ,Subjektivierung ' führe. Der Einzelne wähle nun zunehmend selbst seine Religion, stelle sie aus Elementen unterschiedlicher religiöser Traditionen zusammen, sie sei ihm aber nicht mehr institutionell vorgegeben.

In diesen drei Teilthesen weisen die verschiedenen Vertreter der Individualisierungstheorie auf dem religionssoziologischen Feld - und schon allein dies sollte unsere Individualisierungstheoretiker stutzig machen - eine erstaunliche Übereinstimmung auf. Diese Übereinstimmung können wir hier nicht in extenso vorführen; wir begnügen uns mit zwei Zitaten aus den Veröffentlichungen eines der beiden Replizienten, von Michael Krüggeler. Krüggeler schreibt: „Die hier vertretene These lautet denn auch, dass im Gefolge struktureller Änderungen nicht ein Verlust von Religion - als was die Ent- 
wicklung von den Kirchen gerne wahrgenommen wird - stattfindet, sondern eine Neustrukturierung des Religionssystems und eine Änderung der Äußerungsformen von Religion. (...) In dem Maße, in dem strukturelle Individualisierung institutionelle Verbindlichkeiten zugunsten individueller Entscheidungsspielräume auflöst, wird die kirchlich institutionalisierte Religiosität ersetzt durch eine Tendenz zum religiösen Synkretismus “ (Krüggeler/Voll 1993: 43f.). „Religiöse Orientierungen (...) müssen im Prinzip vom Einzelnen selbst definiert und ausgewählt werden “ (Krüggeler 1993: 93). An die Stelle der Säkularisierungsthese ist damit die Individualisierungsthese getreten. Angesichts dessen liegt es nahe, die behaupteten Zusammenhänge zwischen De-Institutionalisierung der Religion und der Tendenz zu ihrer Synkretisierung und Individualisierung einer empirischen Überprüfung zu unterziehen. Dies haben wir in unserem Artikel getan. Das Ergebnis unserer Untersuchung lautet - kurz zusammengefasst -, dass die Zuwendung zu außerchristlichen und synkretistischen Religionsformen nicht mit einem höheren Grad an Individualisierung verbunden ist.

3. Die Frage, wie Religion definiert werden sollte, begleitet die Religionssoziologie seit ihren Anfängen. Es besteht weitgehende Einigkeit darüber, dass der Religionsbegriff so weit gefasst sein sollte, dass mit ihm auch neuere, nichtchristliche, wenig institutionalisierte Religionsformen erfasst werden können. Gleichzeitig ist klar, dass sich ein Religionsbegriff nur dann analytisch einsetzen lässt, wenn mit seiner Hilfe unterschiedliche Grade von Religiosität erfasst werden können und wenn es möglich ist, auf seiner Grundlage Religion von NichtReligion zu unterscheiden. Ein Religionsbegriff, der es ausschließt, Teile der Bevölkerung als religionslos zu klassifizieren, ist analytisch wertlos. Es sollte also ein Religionsbegriff vermieden werden, der per definitionem jedem Menschen Religiosität, vielleicht sogar jedem den gleichen Grad an Religiosität zuschreibt, denn mit ihm lässt sich die Verbreitung von Religion in einer gegebenen Gesellschaft nicht messen. Die Frage, ob der von Luckmann unterbreitete und von Wohlrab-Sahr und Krüggeler mit Sympathie aufgenommene Vorschlag, Religion in den subjektiven Systemen letzter Relevanzen zu verorten, dieser Gefahr entgeht, lassen wir hier offen. Eine funktionale Bestimmung von Religion wird die Tendenz zur Universalisierung des definierten Phänomens nie vollständig vermeiden können, denn mit der Angabe eines funktionalen Bezugsproblems von Religion - handele es sich nun um subjektive Letztrelevanzen oder Kontingenzprobleme oder was auch immer - ist ein Bedarf zur Lösung des ausgemachten Problems unterstellt, der dann natürlich auch erfüllt werden muss, und sei es durch funktionale Äquivalente von Religion. Um diesen Schwierigkeiten $\mathrm{zu}$ entkommen, haben wir eine substantielle Religionsdefinition vorgeschlagen, die verschiedene Dimensionen von Religion unterscheidet. Dass wir damit eine enge Operationalisierung des Religionsbegriffes vorgenommen hätten, können wir nicht erkennen. In unseren Definitionsvorschlag sind institutionelle Dimensionen von Religion ebenso einbezogen wie individuelle, christliche ebenso wie außerchristliche und synkretistische. Auch auf den Zusammenhang zwischen okkult-magischen Religionsvorstellungen und neuen außerchristlichen Religionspraktiken und -vorstellungen wie New Age, Zen, Yoga, Edelstein-Therapie usw. sind wir eingegangen. Freilich ist zuzugeben, dass die Indikatoren zur Messung der nicht-institutionalisierten, diffusen Religiosität der Verfeinerung bedürfen.

4. Das Problem, das Wohlrab-Sahr und Krüggeler mit den von uns vorgelegten Analysen haben, scheint an vielen Stellen aber nicht in der Tatsache $\mathrm{zu}$ bestehen, dass die von uns verwendeten Messinstrumente nicht fein genug sind. Es scheint vielmehr darin zu liegen, dass wir uns nicht mit der Analyse institutionalisierter Religion begnügen, sondern uns auch mit nicht-institutionalisierter, diffuser Religion beschäftigen und uns dabei standardisierter Verfahren bedienen. Die Replizienten bezweifeln, dass sich eine solche Form von Religion mit Mitteln der standardisierten Forschung erfassen lässt. Zunächst ist es zweifellos richtig, dass ohne valide Messinstrumente theoretische Thesen empirisch nicht überprüft und soziale Phänomene nicht erfasst werden können. Dies trifft auf die quantitative Forschung ebenso zu wie auf die qualitative. Im Gegensatz zu dieser legt die mit quantitativen Methoden arbeitende Forschung jedoch ihr Messinstrument offen und macht es auf diese Weise kritisierbar und überprüfbar. Außerdem ist die mit standardisierten Verfahren arbeitende Forschung aufgrund der Verfeinerung der Erhebungsmethoden, der Entwicklung multivariater Analyseverfahren und loglinearer Modelle heute in der Lage, nicht nur oberflächliche Meinungen zu bestimmten Themen zu erfassen, sondern Meinungen als Indikatoren für tiefer liegende Einstellungen, für komplexe Wert- und Überzeugungssysteme, ja, wenn man so will, für Deutungsmuster zu behandeln. Gewiss müssen sich die Religionssoziologen, die mit standardisierten Verfahren arbeiten, den Vorwurf gefallen lassen, bisher noch kein ausreichend diffe- 
renziertes Instrumentarium zur Erfassung außerkirchlicher, nicht-institutionalisierter Religiosität entwickelt zu haben. Daraus den Schluss zu ziehen, die standardisierte Umfrageforschung könne solche Religiosität nicht erfassen, hieße jedoch, das Kind mit dem Bade auszuschütten. Auch Soziologen, die mit nicht-standardisierten empirischen Verfahren arbeiten, sollten ein Interesse daran haben, ihre Hypothesen zu verallgemeinern und auf ihre Verallgemeinerbarkeit hin zu testen.

Die generelle Kritik von Wohlrab-Sahr und Krüggeler am Gebrauch quantitativer Methoden ist um so unverständlicher, als sie sich, wenn es ihnen darum geht, unsere Analysen zu kritisieren, selbst auf die standardisierte Umfrageforschung beziehen und uns auffordern, Variablen religiöser Semantik und religiöser Praxis mit solchen der Sozialstruktur zu korrelieren. Dieser Aufforderung können wir uns nur anschließen. An einer anderen Stelle, auf die Wohlrab-Sahr und Krüggeler selbst hinweisen, haben wir eine solche Korrelation selbst vorgenommen. Allerdings kommen wir dabei nicht zu dem Ergebnis, dass Religion ihre sozialstrukturellen Konturen weitgehend verloren hat, sondern dass die unterschiedlichen Merkmale von Religiosität durchaus Zusammenhänge mit sozialstrukturellen Differenzen aufweisen (Pollack/Pickel 1999b: 634ff.). Im Übrigen lassen sich solche Zusammenhänge zwischen Sozialstruktur und Religiosität unschwer auch in der Schweizer Studie „Jede(r) ein Sonderfall“, an der neben Michael Krüggeler und Peter Voll auch andere Autoren mitgearbeitet haben, nachweisen (vgl. Pollack 1997).

5. Teilweise berechtigt ist die Kritik von WohlrabSahr und Krüggeler an der von uns vorgenommenen empirischen Umsetzung des Individualisierungstheorems. Dass sich hier Einwände formulieren lassen, haben wir selbst eingeräumt (Pollack/Pickel 1999a: 469). Unsere Operationalisierung des Individualisierungsbegriffs bedient sich nur eines subjektiven Individualisierungsbegriffs, bewegt sich also lediglich auf der Ebene des Selbstverständnisses der Befragten. Ob der individualistischen Intention der Befragten ein individualisiertes Handeln entspricht, haben wir nicht gemessen. Um diesem Problem zu begegnen, haben wir allerdings den Indikator Selbstbestimmung ergänzt durch eine Reihe weiterer Indikatoren, die in der Lage sind, Individualisierungstendenzen abzubilden (470). Auch wenn damit die Ebene der Selbstaussagen nicht verlassen ist, so haben wir durch die Entwicklung eines solchen breiten Individualisierungsindex unseren Individualisierungsbegriff empirisch doch auf eine solidere Basis gestellt. Es ist durchaus nicht aus- gemacht - darin haben Wohlrab-Sahr und Krüggeler Recht -, dass sich ein Individuum so, wie es vorgibt, auch tatsächlich verhält. Aber die Wahrscheinlichkeit, dass selbstbestimmt gehandelt wird, ist wohl zweifellos bei denen, die das wollen, größer als bei denen, die dieses Handlungsziel für sich von vornherein verneinen.

Dass sich der Grad der Autonomie des Handelns durch die qualitative Rekonstruktion komplexer Entscheidungsprozesse erfassen lässt, wollen wir nun unsererseits kritisch in Frage stellen. Zum einen dürfte Einigkeit darüber bestehen, dass auch bei einer solchen Rekonstruktion von den Intentionen der Handelnden ausgegangen werden müsste, und Intentionen lassen sich gerade durch die Umfrageforschung sehr gut ermitteln. Zum andern wird bei einer qualitativen Einzelfallrekonstruktion gar nichts anderes herauskommen können, als dass die Entscheidung individuell gefallen ist, da die Rekonstruktion ja jeweils für einzelne Individuen erfolgt. Um Individualisierung zu messen, bedürfte es aber gerade eines Kriteriums, das den Prozess der Individualisierung als sozialen Prozess erfasst und es insofern erlaubt, verschiedene Individualisierungsgrade zu unterscheiden.

6. Widersprechen müssen wir auch der Auffassung von Wohlrab-Sahr und Krüggeler, dass es sich bei De-Institutionalisierung und Individualisierung um komplementäre Prozesse handelt. De-Institutionalisierungsprozesse auf dem religiösen Feld, die sich sehr gut empirisch nachweisen lassen, müssen durchaus nicht zwangsläufig Prozesse der religiösen Individualisierung nach sich ziehen. Es kann ebenso sein, dass die De-Institutionalisierung des Religiösen Haltungen religiöser Indifferenz oder der Ablehnung von Religion zur Folge hat. Es ist eine Blindstelle der Individualisierungsthese, dass sie die Möglichkeit zur Einnahme areligiöser oder religiös gleichgültiger Orientierungen nicht erlaubt. Dabei wollen wir durchaus nicht so weit gehen zu behaupten, dass Entkirchlichung nicht auch immer wieder mit Prozessen der religiösen Individualisierung verbunden sein kann. Dominant scheint uns aber der Trend hin zu einer stärkeren Distanzierung von Religion und Kirche in allen ihren Dimensionen zu sein, ihren christlichen, kirchlichen, aber auch ihren außerchristlichen und diffusen, nicht-institutionalisierten Formen. In einem so verstandenen Prozess der Säkularisierung dürften - das ist richtig - auch Prozesse der religiösen Individualisierung eingelagert sein, so dass man die Individualisierungsthese auch als einen Teilaspekt der Säkularisierungsthese behandeln könnte. Die Individualisierungsthese in ihrer bisherigen Form behauptet aber einen mehr 
oder weniger zwangsläufigen Zusammenhang zwischen De-Institutionalisierung des Religiösen und religiöser Individualisierung. Es war ein wesentliches Anliegen unserer Studie, diesen Automatismus in Frage zu stellen.

\section{Literatur}

Beck, Ulrich, 1983: Jenseits von Stand und Klasse? Soziale Ungleichheit, gesellschaftliche Individualisierungstendenzen und die Entstehung neuer sozialer Formationen und Identitäten. Soziale Welt, Sonderband 2: 35-74.

Kohli, Martin, 1985: Die Institutionalisierung des Lebenslaufs: Historische Befunde und theoretische Argumente. Kölner Zeitschrift für Soziologie und Sozialpsychologie 37: 1-29.

Krüggeler, Michael, 1993: Inseln der Seligen: Religiöse Orientierungen in der Schweiz, S. 93-132 in: Dubach, Alfred / Campiche, Roland, (Hrsg.): Jede(r) ein Sonderfall? Religion in der Schweiz. Zürich; Basel: NZN Buchverlag.

Krüggeler, Michael / Voll, Peter, 1993: Strukturelle Individualisierung - ein Leitfaden durchs Labyrinth der Empirie, S. 17-49 in: Dubach, Alfred / Campiche, Roland, (Hrsg.): Jede(r) ein Sonderfall? Religion in der Schweiz. Zürich; Basel: NZN Buchverlag.
Pollack, Detlef, 1997: Die Bindungsfähigkeit der Kirchen: Ein Kommentar zur „Sonderfall,-Studie, S. 57-81 in: Dubach, Alfred / Lienemann, Wolfgang, (Hrsg.): Aussicht auf Zukunft: Auf der Suche nach der sozialen Gestalt der Kirchen von morgen; Kommentare zur Studie "Jede(r) ein Sonderfall? Religion in der Schweiz", Band 2. Zürich; Basel: NZN Buchverlag.

Pollack, Detlef / Pickel, Gert, 1999a: Individualisierung und religiöser Wandel in der Bundesrepublik Deutschland. Zeitschrift für Soziologie 28: 465-483.

Pollack, Detlef / Pickel, Gert, 1999b: Individualisierung auf dem religiösen Feld, S. 623-642 in: Honegger, Claudia / Hradil, Stefan / Traxler, Franz, (Hrsg.): Grenzenlose Gesellschaft? Verhandlungen des 29. Kongresses der Deutschen Gesellschaft für Soziologie, des 16. Kongresses der Österreichischen Gesellschaft für Soziologie, des 11. Kongresses der Schweizerischen Gesellschaft für Soziologie in Freiburg i.Br. 1998. Opladen: Leske + Budrich

Tyrell, Hartmann, 1996: Religionssoziologie. Geschichte und Gesellschaft 22: 428-457.

Wohlrab-Sahr, Monika, 1997: Individualisierung: Differenzierungsprozess und Zurechnungsmodus.S. 23-36 in: Beck, Ulrich / Sopp, Peter, (Hrsg.): Individualisierung und Integration: Neue Konfliktlinien und neuer Integrationsmodus? Opladen: Leske + Budrich. 